\title{
La carte de Grèce de Rigas et le nom de la Grèce*
}

Jean-Yves Guiomar et Marie-Thérèse Lorain

\section{(2) OpenEdition}

\section{Journals}

\section{Édition électronique}

URL : https://journals.openedition.org/ahrf/106

DOI : 10.4000/ahrf.106

ISSN : 1952-403X

Éditeur :

Armand Colin, Société des études robespierristes

Édition imprimée

Date de publication : 1 mars 2000

Pagination : 101-125

ISSN : 0003-4436

Référence électronique

Jean-Yves Guiomar et Marie-Thérèse Lorain, "La carte de Grèce de Rigas et le nom de la Grèce* »,

Annales historiques de la Révolution française [En ligne], 319 I janvier-mars 2000, mis en ligne le 11 mai 2006, consulté le 22 avril 2022. URL : http://journals.openedition.org/ahrf/106 ; DOI : https://doi.org/ 10.4000/ahrf.106

Ce document a été généré automatiquement le 22 avril 2022

Tous droits réservés 


\title{
La carte de Grèce de Rigas et le nom de la Grèce*
}

\author{
Jean-Yves Guiomar et Marie-Thérèse Lorain
}

1 En 1998, la Grèce a commémoré avec éclat le bicentenaire de l'exécution par les Ottomans, le 25 juin 1798, de Rigas, premier martyr de la cause protonationale grecque $^{1}$. Comme l'avait montré le très riche colloque d'Athènes de 1987 sur La Révolution française et l'hellénisme moderne $e^{2}$, le mouvement qui a abouti en 1830 à l'indépendance de la Grèce résulte d'une prise de conscience qui monte en puissance dans les trois dernières décennies du xviIIe siècle. Par sa formation et sa vie, Rigas en témoigne particulièrement.

2 Ce qui nous retiendra ici, c'est le fait que, parmi les nombreux documents que Rigas avait réalisés avec ses amis de la bourgeoisie grecque de Vienne [l'un des hauts lieux de la conscience hellénique à cette époque ${ }^{3}$ ] afin de préparer un soulèvement en Grèce, il y a une carte, qui est un véritable manifeste politique. C'est à notre connaissance un cas unique : exprimer par une carte un programme de libération ${ }^{4}$. C'est ce programme que nous allons analyser. 


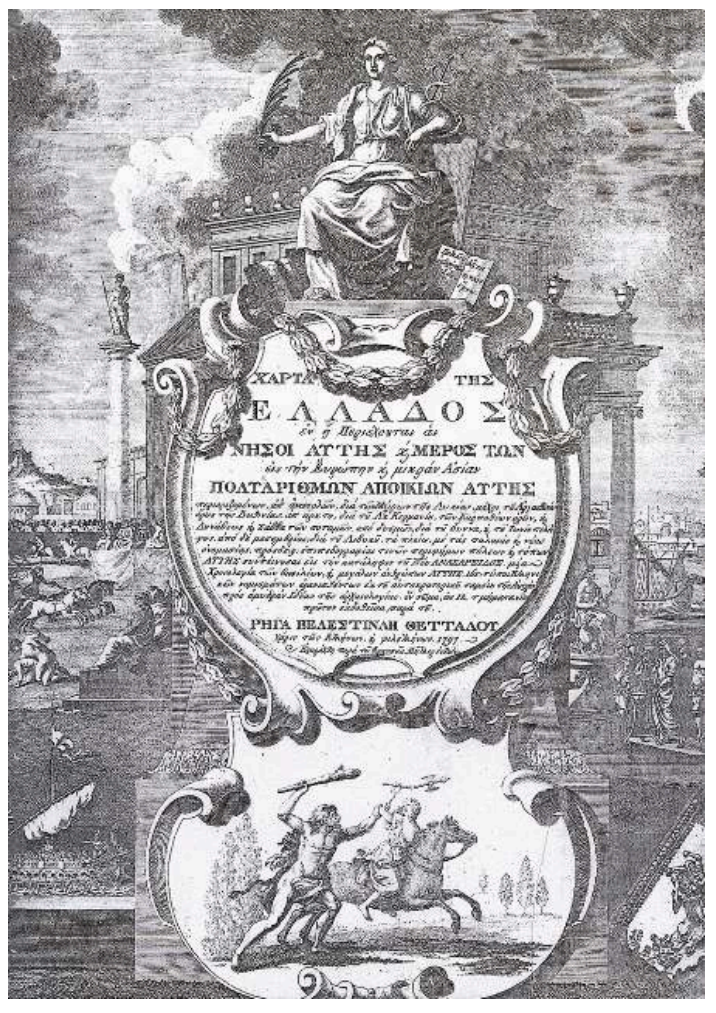

Cartouche de la carte de Rigas (Charta tès Ellados)

Présentation

Au milieu du xvie siècle (sans doute avant 1543), le Grec Nicolaos Sophianos réalise la première carte de Grèce dessinée par un Grec, Totius Graeciae Descriptio. Comme son nom l'indique, cette carte est en latin, mais le cartouche comporte le mot Ellas. 250 ans plus tard, Rigas est le deuxième Grec à réaliser une carte de la Grèce, qui cette fois est en grec, et qui s'intitule Charta tès Ellados (Rigas met le mot carte au féminin, ce qui est inhabituel en grec).

4 L'aire de la carte de Sophianos est nettement plus vaste que celle de la Grèce propre, qui comprenait le Péloponnèse, l'Attique, la Thessalie, l'Épire et les îles de la mer Égée et Ioniennes ainsi que les colonies. Celle de Rigas est la même, mais y ajoute les pays au nord du Danube.

Dans sa communication au colloque de Delphes ${ }^{5}$, qu'il a très aimablement mise à notre disposition, Georges Tolias distingue trois temps : celui de Sophianos, celui de Rigas, et un troisième dans la période 1875-1915, concernant les cartes de Grèce de l'Association pour la diffusion des lettres grecques. Ce troisième temps correspond à la poussée maximale de la Grande Idée (Megali Idea, rêve de reconstitution de l'Empire byzantin, en réalité, rêve de l'unification des Balkans sous la domination des Grecs), dont la carte de Rigas est une expression en termes révolutionnaires ${ }^{6}$. Les trois phases correspondent à des mouvements de diaspora, trait dont nous verrons l'importance.

Qui est Rigas? On le trouve nommé Rigas (ou Rhigas) Velestinlis, de son village natal de Velestino (Thessalie), ou Rigas Pheraios, du nom de la ville antique de Phères, située sur l'emplacement de Velestino. L'un des neuf plans de villes qu'il a mis sur sa carte est celui de Phères (le seul plan qu'il ait dessiné lui-même, juste à côté du cartouche et audessus de celui d'Athènes). 
7 Rigas est donc un Thessalien, né vers 1757 (on en fait parfois un Valaque) ${ }^{7}$. Après avoir enseigné dans sa région natale, il se rend à Constantinople, s'occupe de commerce, apprend le français, l'allemand et l'italien, et entre dans les milieux phanariotes. En 1785, il est au service d'Alexandre Ypsilanti et séjourne à Iassi, puis à Bucarest. En août 1796, il s'installe à Vienne et y demeure jusqu'à son départ pour Trieste en décembre 1797. C'est dans cette période qu'il réalise sa carte. Même si elle a bénéficié de travaux préparatoires dans les Principautés (à Iassi et Bucarest, Rigas avait travaillé à des cartes de Moldavie et de Valachie), elle a cependant été faite très vite. Ce qui peut expliquer la précipitation de ses travaux et son départ de Vienne, c'est la présence française en Italie et dans les îles Ioniennes (les espoirs grecs entretenus depuis les années 1770 par les Russes, ainsi que par les Habsbourg, ont été déçus et sont en train de se reporter sur les Français).

Rigas quitte Vienne pour aller en Grèce avec un matériel destiné à préparer un soulèvement [carte, constitution, manuel militaire, portrait d'Alexandre, Thourios $\left(^{8}\right)$ ]. Dénoncé à Trieste, il est arrêté par les Autrichiens qui en mai 1798 le remettent aux autorités ottomanes de Belgrade. Dans la nuit du 24 au 25 juin, Rigas et six de ses compagnons sont exécutés et jetés dans le Danube. L'événement est connu - le Moniteur parle de l'arrestation ${ }^{9}$-, Rigas est le premier martyr de la cause grecque moderne, et reconnu comme tel jusqu'à nos jours ${ }^{10}$.

9 Le plan de Constantinople (première feuille, sortie à Vienne en août 1796), la Thessalie et les Principautés danubiennes, surtout la Valachie autour de Bucarest, sont les points forts de la carte, liés à sa propre vie. Deux de ces espaces, Constantinople et la Valachie, sont excentrés par rapport à la Grèce proprement dite, celle de l'Antiquité, qui par les noms figurant sur la carte est son thème central.

Cette importance donnée à des espaces périphériques correspond à l'idéologie des Phanariotes. Ils vivent au Phanar, quartier grec d'Istanbul, occupent le poste de grand drogman (interprète) de la Porte dès la fin du xvire siècle, jouant ainsi un rôle clef dans la politique extérieure de l'Empire ottoman, sont fortement liés au patriarcat qui règne sur l'orthodoxie dans l'Empire, et à partir du début xviIIe sont hospodars (gouverneurs) des Principautés danubiennes - Valachie et Moldavie. Jusqu'aux années 1810, douze familles se partagent l'hospodariat, dont neuf grecques, deux roumaines et une albanaise. Ces princes phanariotes sont liés à la Russie et mènent un double jeu, servant les projets grecs des tsars, surtout CatherineII (ses petits-fils sont prénommés Alexandre et Constantin) dans les années 1770-1790. La Russie caresse alors l'idée d'un empire grec orthodoxe prenant la suite des Ottomans. C'est le cadre de la formidable extension donnée par Rigas à l'espace hellénique sur lequel il envisage d'établir un État. Cet État déborde même l'aire des Phanariotes, puisqu'il comprend la Bosnie, la Serbie et l'Albanie.

11 Pour comprendre les buts de Rigas et introduire à l'analyse de sa carte, il faut dire un mot de son projet d'État d'après sa constitution, qui est le décalque des constitutions françaises de 1793 et $1795^{11}$. C'est un État hellénique (terme sur lequel on reviendra), dans lequel tous les citoyens sont égaux indépendamment de leur appartenance ethnique, de leur langue, de leur religion. C'est un État unitaire, un et indivisible. C'est l'idée d'un État qui comprend tout ce qu'on appelle les Balkans plus la future Roumanie. Cependant l'élément grec y est dominant, aussi bien quant à l'appartenance ethnique (tout Grec, où qu'il vive, est citoyen potentiel) et à la langue, qui sera celle de l'État et de la culture ${ }^{12}$. Aussi l'essentiel de cette carte, le cœur de son message, est le fait qu'elle 
soit en grec et privilégie massivement le vocabulaire grec, les noms propres grecs et l'histoire grecque antique, la seule histoire - avec quelques éléments d'histoire romaine - présente sur la carte (avec une exception notable la défaite des Serbes à la bataille du champ des Merles au Kossovo en 1389, transformée en victoire avec la mention du tombeau du sultan Mourad, parce que le chef des ennemis y a été tué).

Le but de Rigas, c'est de transformer cet espace en territoire. Et cette transformation, il la tente par le détour du passé, du recours à l'Antiquité grecque glorieuse. Nous ne pouvons donner ici que de brèves indications sur ce recours à l'Antiquité, en soulignant que pour l'essentiel elle est due aux Lumières françaises, notamment dans les Principautés danubiennes, où l'enseignement de la langue et de la littérature française se développe fortement dans la seconde moitié du xviIIe siècle.

Mais surtout il y a la cartographie française. L'association ancien-moderne est un trait capital du xviIIe siècle français (cela avait commencé au xvıIe et même au xvie). Comme l'a souligné Mireille Pastoureau, d'Anville considère qu'une bonne carte moderne est une étape vers une bonne carte historique ${ }^{13}$. Comme nous le verrons, l'original de la carte de Rigas est à chercher chez Guillaume Delisle. Mais plus près de Rigas, il y a une source directe et essentielle : c'est l'édition en 1788 du Voyage du jeune Anacharsis, de l'abbé Barthélemy, l'un des plus gros succès de la fin du siècle et du xixe ${ }^{14}$, dont Rigas à Vienne traduit l'un des volumes (sept en tout). Officiellement, la carte est faite pour illustrer cet ouvrage (c'est indiqué sur le cartouche). Rappelons que c'est le voyage d'un Scythe (donc un Barbare), Anacharsis, en compagnie d'un noble thébain devenu esclave à la suite d'une guerre, mais affranchi; ils partent en 363 et reviennent en 338, date remarquable (Chéronée, victoire de Philippe sur Athènes-Thèbes, fin de la Grèce propre indépendante). L'itinéraire démarre en Crimée, passe par Constantinople, la Thessalie, l'Épire, l'Attique, le Péloponnèse et l'Asie Mineure (donc exclut les Principautés, la Macédoine, et les régions à l'ouest et au sud de Belgrade). Comme illustrateur en cartes du Voyage, il y a Barbié du Bocage, qui par son travail cartographique joue un rôle clef dans l'émergence de la Grèce ancienne-moderne ${ }^{15}$.

14 La carte de Rigas n'a guère joué de rôle après sa publication, car elle a été victime de la répression de 1798. Pourtant, les Grecs de Vienne y tenaient puisque Antime Gazi, desservant d'une paroisse orthodoxe de Vienne, en donne en 1800 une version simplifiée, mais avec la Grande Grèce et Chypre (G. Tolias indique que c'est la première fois que Chypre figure sur une carte de Grèce). Ce qui est curieux, c'est que Barbié, très lié à des géographes grecs [dont Daniel Philippidès ${ }^{16}$ ] a eu connaissance de la carte d'Antime $\mathrm{Gazi}^{17}$, qui en est la copie, mais pas de celle de Rigas.

Résumons les éléments de la question.

16 1) Une conscience hellénique de plus en plus vive depuis les années 1770. D’abord liée aux Russes et aux Autrichiens, elle se tourne vers les Français, surtout lorsqu'ils prennent pied dans l'espace grec à l'été 1797, avec l'expédition envoyée de Venise par Bonaparte pour prendre les îles Ioniennes.

17 2) Une forte présence des Grecs, mais à la périphérie de la Grèce propre. Constantinople, Bucarest, Iassi, Vienne, Trieste, Odessa, Marseille, etc. Il y a eu une énorme émigration de Thessalie et de Macédoine au cours des xvire-XviIIe siècles. Constantinople à la fin du xviIIe siècle a 700000 habitants, dont seulement 200000 Turcs. À cette époque Athènes a entre 6000 et 8000 habitants. Salonique n'aura 72000 habitants qu'au milieu du xixe. Ces Grecs diasporiques s'enrichissent (la marine de 
commerce se développe fortement ${ }^{18}$, et l'inspiration intellectuelle puis politique française avive leur dessein de s'émanciper.

3) Quel sera le cadre de cette émancipation? Au temps de Rigas, c'est celui de la conscience phanariote, c'est-à-dire celui de l'Empire ottoman qui n'est donc pas répudié mais voué à passer sous la direction des Grecs. Car les autres, Slaves et Roumains, sont peu avancés et alors peu connus en Europe occidentale (mais au moment où Rigas conçoit son projet d'État balkanique, la situation est en passe de changer : les Serbes vont entrer en insurrection en 1804 et au début du xixe siècle les Phanariotes perdent la direction des Principautés danubiennes au profit d'autochtones, sous contrôle russe).

Ce que cherche donc Rigas, c'est à signifier et à spécifier comme hellénique un espace qui comprend bien d'autres éléments. Voyons comment la carte traduit ce projet.

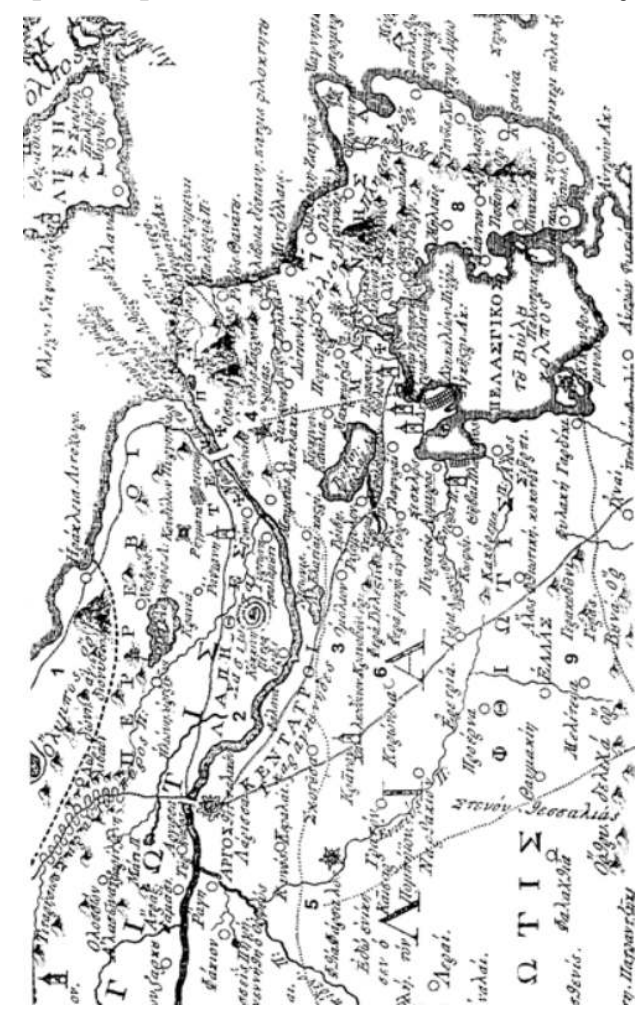

Carte de Rigas (dimensions réelles) - Partie centrale de la feuille 5 : l'ouest de la Thessalie

1. L'Olympe - 2. Les Lapithes - 3. Les Centaures (avec le nom des Karagounes) - 4. L'Ossa (avec la vallée du Tempé au-dessus) - 5. Pharsale (en dessous : " où César a vaincu Pompée ») - 6. PhéraVelestinos - 7. Le Pélion - 8. Mileai (ville natale de Gazi) - 9. La localité de Hellas

(N.B. Les neuf points sont sélectionnés par les auteurs de l'article et n'ont qu'une valeur illustrative.)

Analyse de la carte

Cette carte est un monument de 4 mètres carrés, divisée en douze feuilles ${ }^{19}$. Elle se présente comme profuse et confuse. Son étude géographique et historique déconcerte mais permet de passer à une lecture au second degré et d'avancer une problématique. On vient de dire que Rigas veut unifier ce qui ne l'est pas, et il apparaît en effet que avec cette carte, exceptionnelle par sa surcharge, Rigas veut démontrer que cet espace est homogène. Notre travail sera donc de "décortiquer " ce riche ensemble pour dégager les procédés qui veulent convaincre, et la volonté politique qui est derrière. Nous le ferons à travers cinq éclairages. 
Les éléments généraux de la carte

Si Rigas a pu faire en six mois un tel travail, c'est qu'il a pris comme matrice deux cartes de Guillaume Delisle ${ }^{20}$, intitulées Graeciae Antiquae tabula nova septentrionalis et meridionalis (1707) ${ }^{21}$. La simple comparaison des cartes de Delisle et de celle de Rigas le montre amplement ${ }^{22}$ et Barbié du Bocage nous le confirme. Dans un article du Magasin encyclopédique de 1801, il présente avec sympathie au public français les travaux des géographes grecs ${ }^{23}$ et il écrit : « (...) En géographie, ils ont déjà fait plus que traduire des livres, ils ont traduit des cartes... ». On y « voit exprimé tout ce que fournit la Géographie de Meletius ${ }^{24}$. Malheureusement le plan de cette carte est devenu un peu ancien : c'est celui de la carte de Delisle auquel on a ajouté les parties septentrionales de la Turquie. » Sa phrase s'applique à l'œuvre de Rigas par Gazi interposé. Retenons donc les aides qu'il prête à Rigas: Delisle et la Géographie de Meletius, que Barbié posssède dans sa bibliothèque. Quant à nous, nos fils conducteurs sont Meletius, l'abbé Barthélemy, le dictionnaire de Bruzen de La Martinière ${ }^{25}$, et plus encore Delisle lui-même.

Puisque traducteurs il y a, cette fonction implique liberté. Rigas puise à pleines mains mais pour servir ses propres buts. D'où quelques remarques.

Premier point. La carte de Delisle indique un grand nombre de peuples anciens, que Rigas reprend tous, soit un total de 159 (122 sur Delisle, plus 37 pour les feuilles du haut). Cette implantation cartographique permet mieux qu'un commentaire nourri de littérature ancienne de les juxtaposer à égalité, sans souci de leur réputation de civilisés ou de barbares (au sens que les Grecs donnent au mot). Rigas utilise cependant souvent, en plus, le cadre administratif moderne.

Deuxième point. Rigas agrandit Delisle antique en latitude et lui fait gagner trois degrés vers le nord, ce qui étend l'espace qu'il veut signifier comme hellénique. Sa longitude est celle de la Grèce moderne de Delisle.

Troisième point. Ses intentions sont annoncées dans le cartouche. Pourquoi l'a-t-il partiellement modifié ? Ceux des cartes de la fin du xviıe siècle montrent une Grèce esclave et enchaînée ${ }^{26}$, celle de Delisle donne une Grèce plus glorieuse, personnifiée sous les traits de la Science (Epistémé) qui présente ses villes, ses flottes, signes de petits États indépendants, mais aussi ses jeux, lieux de rassemblement et de paix. Rigas figure Deucalion et Pyrrha, héros de la région de Phères, et surtout, à la place des deux philosophes de Delisle, il met Héraklès, brandissant sa massue pour massacrer Hippolité, reine des Amazones. Ce combat mythique se situe en Cappadoce, illustration de l'hellénisme en lutte contre les peuples de l'Orient, idée constante de la carte.

L'extension du territoire mentionné sur le cartouche va jusqu'à l'Unna, le Danube et la Save, « la Grèce et une partie de ses nombreuses colonies ». Rigas utilise le mot apoikia. C'est un mot que l'abbé Barthélemy avait longuement commentée ${ }^{27}$, à propos d'une Grèce pauvre, qui avait besoin d'espace - ce que Rigas actualise au maximum dans sa visée phanariote. Apoikia suggère des États indépendants, différents des "clérouquies ", les colonies de l'empire athénien.

Quatrième point. La carte est bordée, en haut et en bas, d'une liste de noms de souverains. C'est un subtil ajout personnel de Rigas, qui comporte 270 noms. 114 concernent la Grèce indépendante, dans l'ordre alphabétique: héros mythiques, écrivains, gloires militaires (sans oublier Jason, tyran de Phères). Ils sont intrinsèquement liés à la carte: la plupart se retrouvent dans les phrases de commentaires qui la parsèment ${ }^{28}$. Puis, comme le dit Rigas sur la carte, viennent « ceux 
qui ont effectivement régné sur la Grèce »: Alexandre et les quatorze Ptolémées, la dernière étant Cléopâtre. C'est a priori surprenant, puisque seul le fondateur de cette dynastie qui a régné sur l'Égypte est Macédonien. Puis avec une croix latine il introduit Rome, mais seulement avec Auguste, en -31: la conquête romaine est escamotée. Trente-six empereurs romains succèdent à Auguste. L'empire d'Orient puis l'empire byzantin en présentent quatre-vingt-un. On continue avec le Croissant, après une ligne qui évoque le jour de deuil des Grecs à propos du sultan Méhémet, «celui qui a ravagé la Ville » en 1453. Il est suivi de vingt-trois sultans jusqu'à Sélim III, le contemporain de Rigas.

La phrase que nous venons de citer est reprise en écho triste sur le plan de Constantinople. Sous le Lion, qui représente la puissance ottomane comme le faisceau d'armes et le turban, il y a une pièce à l'effigie de Constantin Paléologue, le dernier empereur grec, puis un mot : «Et nous fûmes asservis ».

Nous avons dit à propos du cartouche et du cadre : «les intentions sont annoncées ». Elles nous ont conduit à un message politique aussi fort qu'il est implicite, et masqué par la profusion de la carte.

La Thessalie

31 Ce n'est pas la région la plus célèbre de la Grèce dans nos souvenirs de l'Antiquité. Mais Rigas est thessalien et il nous offre une Thessalie porteuse de germes : il y place un maximum d'informations historiques et mythologiques ${ }^{29}$, pour mettre en évidence le lien indissoluble entre un passé glorieux et un présent à transformer en continuité avec l'ancien.

32 Elle est bien délimitée, comme chez Delisle. Elle couvre les deux-tiers de la feuille 5 (qui est en position centrale sur la carte), avec son cadre de montagnes : le Pinde, l'Olympe, le Pélion, l'œta, et la vallée paradisiaque du Tempé.

33 Elle rassemble 14 peuples, qui permettent de retrouver les strates de la longue histoire qui caractérise ce pays. Déjà Pline avait remarqué que la Thessalie changeait constamment de nom "selon les rois qui la gouvernent ». La Martinière confirme : «Aux Thessaliens on donne le nom de Centaures, puis une succession de noms: Æmonia (Hémonie) quand elle est envahie par les Pélasges, Hellas, Argos, Dryopia ». Or tous ces noms sont là sur la carte - encore une fois comme à égalité -, évocateurs du soubassement légendaire grec.

Rigas fait sortir de sa Thessalie un passé mythologique/historique : les Centaures et les Lapithes ; deux généalogies qui culminent sur Hellen et Hellas ; les Argonautes, que Jason emmène chercher la toison d'or en Colchide: encore un symbole de l'hellénisme conquérant et mythique, puisque Orphée est l'un des rameurs de la barque des Argonautes ; la patrie d'Achille, roi des Myrmidons, est en Achaïe, façon de référer à la guerre de Troie ; Léonidas aux Thermopyles, bataille des guerres médiques, renforcée par un plan tiré du Jeune Anacharsis (mais sans citer Barbié) ; Philippe est là aussi, Rigas indique Méthone "où Philippe reçut dans l'œil la flèche qui le rendit borgne ", sans faire allusion au siège qu'il fit de cette ville en -353 ; la bataille de Pharsale « où César a abattu Pompée » (puisque c'est une bataille entre Romains, Rigas peut la figurer).

Il évoque aussi le présent : au pied du Pélion, il met les Karagounes, peuple moderne, sédentaire, grécophone, en contact avec les bergers valaques plus nomades. Au pied de l'œta, il met les Lidoriki et les Krabari, qui sont aussi des données actuelles. Il y a une 
référence aux hauts lieux de l'orthodoxie : les monastères des Météores ${ }^{30}$ et du mont Athos.

Un Thessalien se devait d'être complet sur sa propre région, dûment présentée comme matrice de la Grèce : assurément, il l'a été. Avec même une pointe de patriotisme local, il fait figurer dans la liste du haut de la carte les deux Jason, celui des Argonautes et le tyran de Phères qui, quarante ans avant Philippe, a voulu unir les Grecs contre les Perses.

La Macédoine et le problème des montagnes

37 Ces montagnes de la Macédoine (en Péonie) et de la Thrace (le Balkan) sont un endroit stratégique essentiel pour Rigas, car elles l'obligent à affronter le difficile problème de la jonction entre l'espace grec et l'espace danubien. Les cartes imposaient depuis le xve siècle une Catena Mundi, chaîne continue barrant l'espace de la côte dalmate à la mer Noire, donnant l'impression d'une puissante coupure par rapport au reste de l'Europe ${ }^{31}$. Avec ses noms de l'Antiquité, cette chaîne figure sur les cartes de Sophianos et de Delisle. À partir d'un nœud orographique (le Scardus), la branche orientale va vers la mer Noire par les monts Orbelus, Scomius, Hemus (avec en annexe vers le sud les monts Berthiscos, le Pangée, l'énorme Rhodope). La branche occidentale arrive à la côte dalmate par les monts Krôton et Borée.

Rigas reprend ce schéma, mais d'une manière plus confuse et significative, car il lui faut supprimer cette barrière. Il va morceler la chaîne infranchissable à l'aide de noms différents, et élargir les vallées. Il est aidé en cela par les tendances qui émergent dans la géographie du temps, celle des bassins de Buache ${ }^{32}$. Allant dans le même sens, La Martinière exprime en 1768 des positions théoriques intéressantes. Voyons brièvement deux de ses citations :

L'Hemus: «Les modernes ne conviennent pas sur le nom que porte actuellement cette montagne (de Thrace). Laonic la nomme Prasovo, les Italiens, d'après Pinet, Catena del mondo et Monte Argentaro. Les Turcs l'appellent le Balkan, et les Esclavons Cumowicz. Le même Pinet, dans sa traduction de Pline, dit aussi monts de Castegnas ». De sorte qu'à l'unique Hemus de Delisle se substituent quatre noms alignés par Rigas : l'Hemus antique, le Kostegnas de Pinet, le Balkan des Turcs et le Kumanitza des Esclavons. La barrière commence à s'ouvrir !

L'Orbelius :La Martinière donne la localisation traditionnelle « au nord de la Macédoine, entre Péonie au midi, Scordisques au nord, Dentélètes à l'orient ", et en propose une seconde « ou pour s'exprimer de façon moins sujette aux révolutions, entre l'Axius au couchant, le Strymon au levant, à l'orient d'Uscupia ». Le terme de révolution fait ici référence aux débats entre cartographes classiques, qui établissent la topographie cartographique à partir des lectures de Ptolémée, Hérodote, Strabon et autres, et les efforts modernes pour établir un critère de fixité géographique, qui serait donné par les fleuves comme facteurs naturels (d'où l'importance de la théorie de Buache) ) $^{33}$. C'est bien ce que feront Humboldt et Ritter. En somme, le grand intuitif qu'était Rigas avait à sa manière anticipé la connaissance qu'apportera le xixe siècle

41 Rappelons quelques jalons de la connaissance de l'orographie de la région, en nous référant à la Géographie universelle de Vidal de la Blache (tome VII, 2e partie). Le nom moderne de Balkan se trouve déjà en 1757 dans le Grand Atlas de Robert de Vaugondy. Gatterer, historien et géographe, précurseur de l'école allemande moderne, est l'auteur d'un Traité de géographie, paru en 1775 à Göttingen, où il cherchait dans la géographie 
physique les divisions naturelles du globe, particulièrement dans les lignes du relief. Il obtint une "péninsule pyrénéenne ", une "alpine ", une pour les pays au nord et au sud de l'Hemus. C'est le géographe allemand Zeune qui le premier, en 1808, employa l'expression "péninsule des Balkans ", dont il détache un Karpatenland, division qui ne sera pas retenue par la suite. En 1828, les Russes s'y aventurent pour la première fois ; en 1840, Ami Boué en commence l'étude scientifique; en 1867-1869, le voyageur cartographe Guillaume Lejean ${ }^{34}$ mène trois campagnes; de 1860 à 1881, l'Autrichien Kanit ${ }^{35}$, son ami plus heureux, en fait dix-huit, pour constater qu'il n'y pas de chaîne centrale, mais un axe formé par les deux vallées de la Morava et du Vardar (ex-Axius), où l'on peut faire passer un chemin de fer. À la fin du siècle, on passera du Balkan, montagne au sud du Danube, aux Balkans, désignation de l'ensemble de la péninsule ${ }^{36}$.

42 Après la géographie physique qui a tendance à se moderniser, la géographique historique fonctionne à plein chez Rigas dans cette zone difficile et pourtant décisive jusqu'à nos jours. Quelle tentation de sortir de ces plaines minuscules et de ces montagnes pour aller vers les vastes plaines du monde danubien! Or Rigas doit absolument remplir cet espace qui fait jonction entre sa Thessalie et sa Valachie. Que lui apporte l'histoire? Un espace aménagé au sud par les Grecs, au nord par les Romains et mille fois envahi dans l'histoire. Après avoir sur le plan géographique tenté de mettre en évidence la continuité, il faut maintenant prouver l'unité. On trouve la clef de sa démarche sur la feuille 5 , en Béotie, dans une phrase-message : "Chéronée où a vécu Plutarque ». Or Chéronée, c'est surtout la victoire de Philippe en 338 sur les Grecs trop tard unis. C'est là qu'Anacharsis rebrousse chemin vers sa Scythie, plutôt que de voir une Grèce écrasée... Rigas préfère donc évoquer Plutarque, l'auteur des Vies parallèles des hommes illustres de la Grèce et de Rome. Rigas fait sa démonstration sur deux plans :

43 -Il aligne une quarantaine de peuples «barbares » qu'il trouve chez Delisle, qui ne semblent pas avoir d'histoire. Sauf un : les Graii, entre Scomios et Pangée, dont il faut apprécier l'intérêt étymologique. Ce nom très rare, et pour cause, dans la littérature grecque (il est tardivement attesté en grec), est pourtant celui qui a été emprunté par les Romains pour donner Graecus, Graecia. Il a subsisté dans toutes les langues romanes $^{37}$. Ni Meletius, dans la préface de sa Géographie, ni les Grecs actuels ne semblent aimer le poids qu'il a pris. Delisle le mettait, Rigas le tolère.

-Il oublie volontairement beaucoup de choses. Les Macédoniens sont-ils des Grecs ? Sa réponse est donnée par le fait qu'il "oublie " Chéronée. Que fait-il de la conquête romaine? Nous avons vu qu'elle n'apparaît pas dans le cadre chronologique. Sur la carte, il faut bien chercher pour en trouver un indice assez "glorieux " pour être acceptable : les quatre «Basileia». Dans la grande Macédoine englobant la moitié de l'Illyrie à l'ouest, la moitié de la Thrace à l'est, et influençant le sud de la péninsule, c'est-à-dire celle des conquêtes de Philippe, jusqu'au malheureux Persée vaincu par Paul Émile en -168 , Rome découpe quatre "Regio ${ }^{38}$. C'est le terme qui figure chez Delisle. Elles deviennent quatre Basileia chez Rigas, avec leur autonomie et chacune une capitale. Peu importe pour lui qu'en -148 une révolte les ramène au rang de provinces romaines. Ces éphémères basileia sont donc une manière délicate, minimale, d'évoquer la conquête, de la suggérer. Rome ensuite franchit le Scardus et l'orbelius, et écrase les Scordisques. Il faudra encore une centaine d'années pour achever sous Auguste la maitrise de cet espace. Mais toutes ces péripéties sont gommées, l'histoire est lissée, la conquête romaine est transformée en un mouvement qui porte l'hellénisme en Mésie, 
grande région de l'Europe ancienne, plaine longue de $900 \mathrm{~km}$ et large de 200, limitée entre Save et Danube au Nord, Scardus/Orbelius au sud, Drin à l'ouest, Pont-Euxin à l'est.

Rigas a ainsi mis en évidence le fait que les Provinces danubiennes font bien partie intégrante du monde hellénique. Il a retourné la conquête romaine du monde grec en expansion de l'hellénisme.

Les principautés danubiennes

Rappelons qu'avec la Thessalie, c'est la deuxième région dont il a une connaissance personnelle et c'est là qu'il a fait l'essentiel de sa « carrière ".

La carte est dominée par l'arc des Carpates, et par un majestueux Danube servi par ses grands affluents: Drave, Save, Tisza, Morava, Jiul, Olt, Argesh, Dimbovitza, Gialomitza, Bouzaion, Pruth. Deux noms ont une taille de caractères qui les met en égale valeur: BLAXIA/VALACHIE, DAXIA/DACIE, l'un actuel, l'autre antique.

Depuis le début du xvine siècle, il y avait en Transylvanie de nombreux travaux sur les origines latines de la Dacie (conquise sous Trajan en 106, elle est la première province abandonnée par Aurélien en 276). Tout le siècle avait connu une forte émigration transylvaine vers les Principautés. À la fin du siècle, une école philologique et historiographique ${ }^{39}$ s'efforçait de mettre en évidence l'unité latine de la future Roumanie. Intégrer l'ensemble Transylvanie-Moldavie-Valachie, en voie de formation commune sur une base de latinité, dans le projet hellénique de Rigas était donc d'une importance capitale.

49 La carte de Rigas donne le foisonnement habituel des peuples, des plus anciens (Theuriskoi, Iazyges, Peukinoi), qui étaient dans l'Antiquité des Daces nomades, aux plus contemporains. Rigas n'a plus ici le soutien méthodique de Guillaume Delisle et il ne peut plus mettre ses pas dans ceux du grand cartographe. Mais il a la chance de pouvoir utiliser la carte que F. Jos. Ruhedorf a publiée en $1788^{40}$, à Vienne, chez Muller, le même graveur que pour la sienne.

Une surprise attend les géographes aux Cartes et Plans de la BNF : une carte autographe de Rigas, carte manuscrite de la Valachie. Nous remercions M. Georges Tolias de nous avoir signalé l'étude de Mme Anna Avraméas, "Carte manuscrite de la Valachie, autographe de Rigas $»^{41}$. Elle y a découvert un relevé à la main de Rigas, simplifié, d'après Ruhedorf: c'est un manuscrit émouvant, parce qu'une étude graphologique permet d'attribuer la paternité de ce calque à Rigas. C'est surtout un manuscrit utile pour comprendre sa façon de travailler. Dans un premier temps, sur un calque, il a parfaitement retracé fleuve et affluents. Dans un deuxième temps, il recopie les dixhuit chiffres romains par lesquels Ruhedorf avait étiqueté sous le nom de districtus les judicetes (circonscriptions administratives) que l'on retrouve dans la Roumanie des xixe et xxe siècles (Mechaedintzi, Romanitzi, Dimbovitza, Ialomitza, etc.).

51 Il faut seulement remarquer qu'à la date où il fait sa carte (1788), Ruhedorf prend ses désirs pour des réalités, et distingue deux cas : dix-huit districts forment une Valachia Austriaca dicta, sur la rive gauche de l'olt, et quatre districts sont dits Valachia Turcica. Certes le traité de Passarowitz (1718) avait donné la rive droite de l'olt - l'olténie - à l'Autriche, mais elle avait été rendue aux Turcs en 1739, cette subdivision avait donc cessé d'être valable. La région turque est confiée par le sultan à l'hospodar Alexandre Ypsilanti.

52 Rigas est donc plus exact que Ruhedorf pour les frontières politiques, mais il utilise la typographie de façon très spéciale. Il fait sur son calque un tableau des noms latins de 
la carte de Ruhedorf (villes, villages, monastères), qu'il numérote, puis il les transpose, en grec, sur sa carte. Il traduit de même les noms de peuples. Il y ajoute ses petites phrases, et surtout il morcelle, en les mettant sur plusieurs lignes, les noms de ces peuples non grecs de la région.L'opération les rend très difficiles à lire et à identifier.

Pourquoi est-il si clair sur son calque et si confus sur sa carte ? Il est évident qu'ici plus qu'ailleurs, il brouille les cartes: la comparaison du calque et de la carte nous offre donc un remarquable éclairage sur son processus d'hellénisation forcée d'un espace non grec $^{42}$.

Ajoutons qu'il "emprunte" aussi à Ruhedorf sa légende, son tracé des routes caravanières, et concluons à l'utilisation intelligente, certes, mais inavouée de Ruhedorf qu'il traduit comme il l'a fait pour Guillaume Delisle, peut-être encore plus méthodiquement.

Rigas n'élimine pas les traces de la présence romaine : il figure la route de Trajan, parallèle à l'olt, montant du Danube vers Rumnix, et les «fondements du pont de trajan" à Séverine. Mais ces éléments sont noyés dans une profusion d'autres éléments : "Sarmigetusa, capitale de Décébale » (vaincu par Trajan), l'administration des hospodars grecs (le "canal construit par Alexandre Ypsilanti») et il indique à Tsernaboda la "patrie de Joseph Mesodaces ${ }^{43}$, homme des lumières ». Il multiplie les routes de caravanes, si essentielles pour l'enrichissement de la bourgeoisie grecque. La seule ville mise en évidence est Bucarest, ville que Rigas peut à juste titre considérer comme un important foyer de diffusion de l'hellénisme. Il mentionne aussi les guerres contemporaines ${ }^{44}$.

Ensuite, on arrive en Thrace, aux confins barbares, au monde inquiétant des steppes. Là, Rigas mêle peuples anciens et modernes. Suivons sa carte, en compagnie de La Martinière.

57 - Anciens : Britolages et Massagètes ("nomades pour Hérodote et Strabon »), Gètes («ancien peuple de Scythie ou Tartarie que Cyrus, Darius et Alexandre tentèrent vainement de dompter ») et Teukinoi (« des Sarmates qui n'ont pas de villes »).

-Moderne :s en Bessarabie, il met des Tartares Budziaks (« environ 30000, réputés les plus méchants qu'on puisse trouver, indomptables aux Turcs»), il met aussi ceux de Dobroujda, «dont les Turcs font des troupes de choc ».

59 Cette nomenclature indifférenciée montre que Rigas là encore unifie artificiellement l'héritage ancien grec et latin, pour donner le sentiment d'une homogénéité d'origine. La magie du verbe de Rigas sera-t-elle capable d'ancrer aux confins d'un monde barbare les preuves de la présence de l'hellénisme? Il en met trois: la mort d'Orphée, l'île d'Achille, "offerte à lui par Thétis, avec un temple à Patrocle, voué depuis à Saint Anastase ", et Tomis, "lieu d'exil d'Ovide». Les cultures grecque et latine sont donc aussi présentes dans ces espaces de l'extrême périphérie, où il y a eu des comptoirs grecs.

Les lieux de commémoration

60 Les lieux de commémoration sont la grande originalité de Rigas. C'est là qu'il y montre le mieux sa volonté de faire partager sa passion, de créer une adhésion affective à son projet révolutionnaire. C'est la fonction des phrases dont nous avons parlé. Nous ne pouvions ici les commenter, nous les avons donc classées dans un tableau placé en annexe. 
61 À noter que pour les batailles, les symboles qu'il utilise sont la massue d'Hercule pour les Grecs contre la pique attribuée aux Perses et aux Turcs. On a aussi des combats navals où la massue s'orne alors d'une ancre. Or on ne trouve aucune trace de combat entre deux massues !

Au total, cette carte semble nous dire: "J'inscris ici mes biens au cadastre » et je cherche à faire plaisir à chacun, à chaque endroit (ville ou région), pour que tous aient la vision concrète de leur rapport propre et ancien à l'hellénisme. Il veut faire participer chacun à la chose globale. Chacun doit trouver sur la carte une trace de sa présence originaire, être une pièce de cet hellénisme que Rigas exalte sur l'immense espace sur lequel il projette de créer un État hellénique, qui unifiera sous la houlette grecque tout ce que le passé a charrié de différent et d'antagoniste, et qui est encore bien présent en son temps.

Né grec en Thessalie, il veut étendre l'hellénisme. Sa chance est d'avoir travaillé en Valachie-Moldavie, ce qui lui a fait prendre conscience d'un vaste territoire où il y avait des populations non grecques mais orthodoxes et opprimées par les Turcs depuis le xve siècle. C'est donc une carte de libération mais aussi d'impérialisme culturel, inscrit dès l'Antiquité.

Essai de synthèse

Repartons du début : cette carte est la première aux temps modernes faite par un Grec. C'est donc non la première réflexion d'un Grec sur l'espace grec (Meletius, Philippidès) mais la première qui tente de se traduire sur une carte. L'entreprise est d'une extrême complexité, car c'est un espace formidablement chargé de significations, par le passé à toutes les époques, par l'étranger, notamment la France et la Russie, et par sa périphérie proche et lointaine.

Rigas n'était pas un « intellectuel » du type des maîtres des Lumières grecques du XviIIe, tels Voulgaris, Mésiodax, Katarzi, ou surtout Adiamantos Coray son contemporain ${ }^{45}$. C'est un imaginatif et un intuitif. L'imagination lui inspire cette carte à la fois somptueuse et désordonnée. Mais l'essentiel est qu'elle est en grec, son moyen linguistisque est adéquat à son objet, le moyen donne le sens du discours même. L'intuition, c'est plus compliqué tout se passe comme s'il avait pressenti que la suite de l'histoire rendait urgente l'affirmation d'une prééminence grecque sur l'espace balkanique, l'affirmation que seuls les Grecs pouvaient donner sens et unité à cet espace qui était celui de la domination ottomane, cadre accepté et repris au compte des Grecs.

66 La suite de l'histoire, c'est la distinction de peuples tels que les Serbes, les Bulgares et les Roumains, peuples et langues que le xvine siècle distinguait fort mal. Rigas connaît les mécontentements serbes et plus encore la rébellion de Pasvan-Oglou dans la région de Vidin (sorte d'amorce d'une future Bulgarie), sans compter les ambitions du tyran Ali Pacha en Épire. Il y aurait donc un aspect « course de vitesse » dans la réalisation de cette carte, dans le parcours personnel de Rigas (sa venue à Vienne) et dans la conjoncture historique.

Tout cela pose frontalement le problème double de l'État qu'il aspirait à créer :

68 -Comment remplir cette Grèce si désespérément vide (en laissant de côté l'Ionie) par rapport à la richesse de sa périphérie?

69 -Comment articuler l'espace grec aux espaces non grecs? Où finit l'espace grec? Son projet d'État hellénique-balkanique sera caduc vingt ans après sa mort, on dirait qu'il a 
vu qu'il fallait absolument mettre en place quelque chose sur le sol, faute de quoi la Grèce ne serait que le Péloponnèse et les environs d'Athènes (en 1830, le nouvel État grec sera à peine plus).

Sur le premier point, l'analyse de la carte a donné de nombreux éléments. Il est clair que l'hellénisation des noms de peuples permet à Rigas de faire l'impasse sur la nature "ethnique » réelle des habitants de la périphérie, au nord-ouest, centre et est. L'analyse a également développé la question complexe de la Macédoine et de la Thrace, ainsi que le difficile problème du lien avec les Principautés danubiennes.

Reprenons la question sur un plan plus général, celui de la dénomination de l'espace sur lequel l'État de Rigas doit s'implanter. La solution, Rigas, mais aussi les Grecs du xIxe siècle, la trouvent dans la terminologie issue d'Hellas: Hellade, hellénisme, hellénique. Cette solution passe donc par le recours au passé lointain, cette Grèce des origines qu'il signifie à partir de sa plus grande extension, celle que lui a donnée Alexandre, mais c'est une extension qui aboutit à la distendre tellement que si le nom de Grecs reste pertinent, celui de Grèce - nom donné par les Romains à partir d'un minuscule peuple excentré et barbare - devient source de difficultés.

C'est ici que le nom d'Ellas ou Hellas et ses dérivés jouent leur rôle clef. Perdu depuis Byzance, Ellas revient au premier plan dans la seconde moitié du xviIIe siècle, et s'imposera comme le nom officiel de la Grèce à l'heure de l'État national. Le dictionnaire de La Martinière signale quatre acceptions pour Ellas: une ville de Thessalie, une région de Thessalie, la Grèce propre, puis la Grèce propre avec ses extensions en Macédoine et en Épire. Rigas n'en retient que deux : la plus restreinte, puisqu'il mentionne non loin de Velestino la localité appelée Hellas, et la plus vaste, puisque c'est le nom qu'il donne à sa carte. Ce faisant, il lègue à la Grèce de 1830 une image de son espace national virtuel, virtualité impliquée par le fait que, situation exceptionnelle en Europe, la Grèce est davantage faite de terres irrédentes que du territoire concédé par les puissances. Signifier que ces terres irrédentes sont bel et bien helléniques, c'est la fonction de ce recours massif au passé, ou plus exactement aux origines.

Il existe un témoignage du caractère en quelque sorte prémonitoire de la carte de Rigas dans le fait qu'entre 1853 et 1875 l'historien grec Constantin Paparrigopoulos, professeur à l'université d'Athènes, publie une monumentale Histoire de la nation hellène (et non grecque), et en donne en français chez Hachette en 1878 l'épilogue sous le titre Histoire de la civilisation hellénique ${ }^{46}$, ouvrages où il cherche à montrer la parfaite continuité de l'hellénisme à travers les siècles, de l'Antiquité à l'époque actuelle en passant par Byzance et le temps de maturation silencieuse sous les Ottomans. Dans sa préface à l'ouvrage de 1878 (ouvrage où il rend hommage à l'action de Rigas), il souligne qu'il est le premier Grec moderne à donner une Histoire grecque (comme Rigas avait été le premier à donner une carte de Grèce en grec). Citons deux passages de cet ouvrage, qui mériterait de longs développements: «Dans sa principale signification, helléniser voulait dire : transmettre la langue grecque à des peuples étrangers et leur imprimer par le moyen de la langue le caractère national des Hellènes. L'hellénisme était le résultat de cette action ${ }^{47}$ ». Et: «En 1790, trois représentants de la nation demandèrent à l'impératrice Catherine de donner pour empereur aux Hellènes son petit-fils Constantin ; ils ne parlèrent plus à titre de Romains ${ }^{48}$ ni même simplement de chrétiens; ils se présentèrent comme des Hellènes, descendants des Athéniens et des Spartiates. $»^{49}$ 
74 Revenons à Rigas, dont nous savons que, par son projet de constitution, il envisageait la création d'un État non ethnique, associant dans une république une et indivisible tous les citoyens vivant sur son territoire, Grecs, Serbes, Roumains, Bulgares, etc., avec certes une prime aux Grecs, à leur conscience d'être le peuple le plus civilisé - le seul, en fait - et le plus glorieux de cet espace, et surtout à la langue grecque [qui joue un rôle central dans la notion d'hellénisme ${ }^{50}$, mais sans que cette république unitaire soit de nature ethnique. Pourtant l'hellénisme de Rigas, comme le montre sa carte, est finalement, autant que politique, historique, culturel et linguistique.

Il y aurait lieu de développer le fait que sa carte, manifeste cartographique de la Grande Idée (même si par son projet Rigas diffère des hommes politiques grecs qui à partir du milieu du XIxe siècle donneront à celle-ci un caractère conquérant, alors que Rigas postule une unité «naturelle » et consentie), apparaît au moment même où la France, à partir de l'été de 1797, s'appelle elle-même «la Grande Nation » ${ }^{51}$. À partir de la campagne d'Italie, ayant précédemment intégré la Belgique et se préparant à faire de même pour la rive gauche du Rhin, la France révolutionnaire du Directoire met en œuvre une conception de la nation en quelque sorte "supranationale ", conception essentiellement politique mais où il existe aussi des éléments historiques (tel le recours massif à la Gaule des origines) et linguistiques (telle la conception que le français est la langue la plus parfaite des langues modernes, l'équivalent du latin dans l'Antiquité). Il est frappant que ni Rigas ni les révolutionnaires français et ceux des pays intégrés ou conquis n'aient vu à quel point ces éléments allaient par la suite prendre partout une importance sans cesse croissante, au point de menacer et parfois de détruire la notion politique de nation, corps de citoyens participant à égalité à la formation de la volonté générale.

*Cet article est issu d'une communication faite le 14 janvier 1999 au séminaire de Daniel Nordman et Marie-Vic Ozouf-Marignier, que nous remercions vivement pour leur accueil, à l'EHESS.

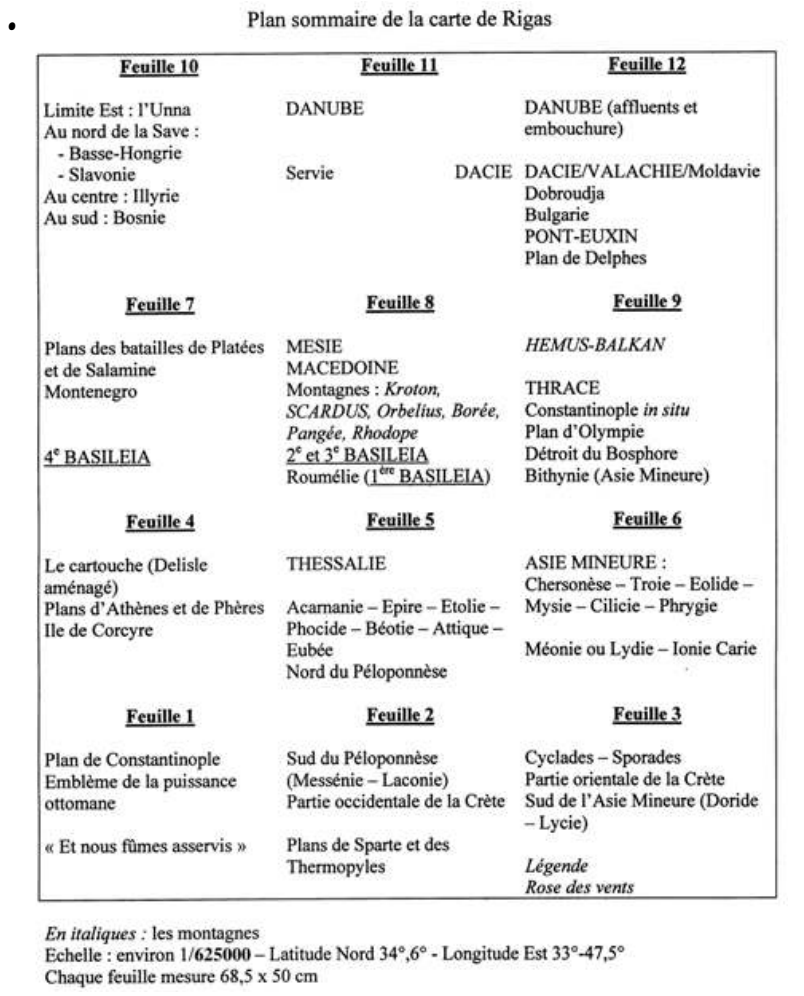



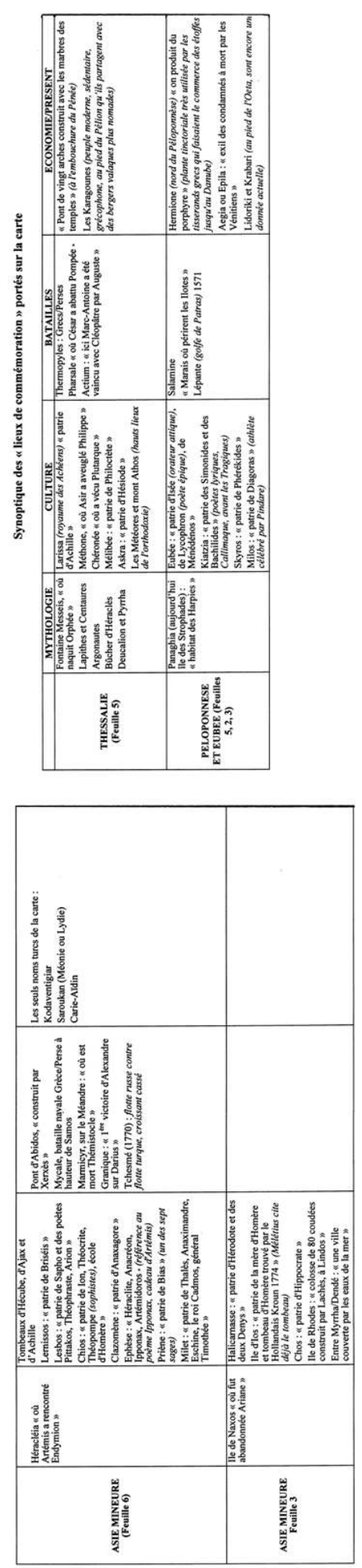


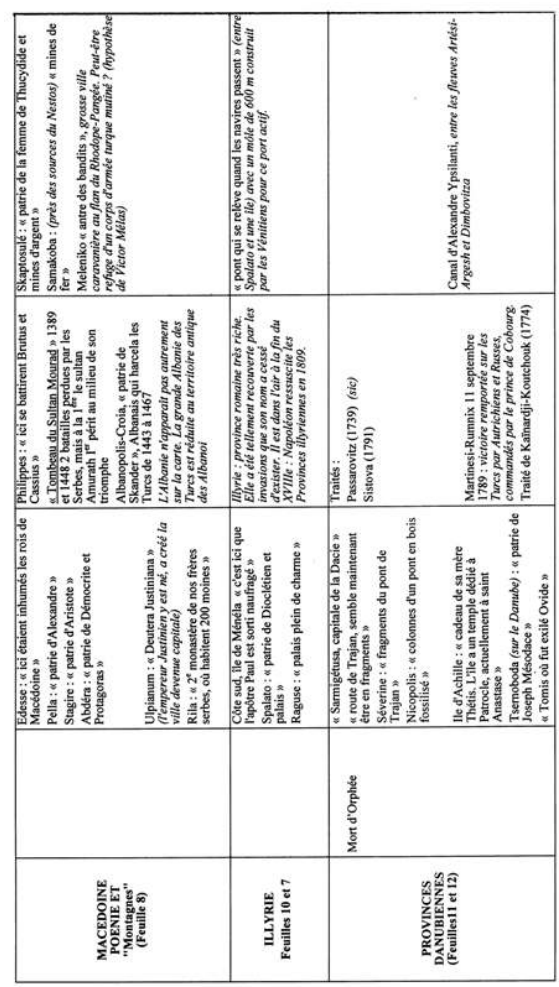

\section{NOTES}

1.De nombreux colloques ont eu lieu, notamment à Velestino (ville natale de Rigas) en 1997, à Delphes en juin 1998, à l'Unesco à Paris en décembre 1998. Cf. infra un compte rendu de certaines de ces rencontres dans la rubrique « Mélanges".

2.Fondation nationale de la Recherche scientifique, Athènes, 1989 (en français).

3.Il existe une belle étude (en grec) de Georges Laïos sur les milieux grecs de Vienne à l'époque du séjour de Rigas, milieux gagnés à la cause de l'émancipation nationale et des idées révolutionnaires françaises, étude parue dans le Deltion tès historikès et ethnologikès etairéias tès Helladès ( Bulletin de la Société historique et ethnographique de Grèce »), pp. 202-270, 1958. Pour ce texte comme pour tous les autres textes en grec auxquels nous avons recouru, une traduction nous a été faite par Vanghelis Karamanès, que nous remercions chaleureusement. Sur les projets d'émancipation grecque avant 1821, et notamment ceux de Rigas, voir aussi A. Manessis, «L'activité et les projets politiques des patriotes grecs dans les Balkans vers la fin du Xvirie siècle ", Balkans Studies, tome III, 1962.

4.Cette carte est aujourd'hui en Grèce l'objet de nombreux travaux, mais il n'en a pas toujours été ainsi. La première étude, française, est un bel article d'Ubicini, « La grande carte de Grèce par Rhigas ", paru dans la Revue de géographie en 1881 (année décisive où la Grèce « récupère » la Thessalie). Rappelons la thèse complémentaire d'Ap. Dascalakis sur Rigas, Les œuvres de Rhigas Velestinlis, Paris, 1937, qui consacre quelques 
développements à la carte. Mentionnons ensuite un article de Georges Laios, dans le Deltion..., pp. 231-286, 1960. Victor Melas lui a consacré en 1973 une étude, reprise à l'Unesco en 1998. Les derniers grands travaux en date sont (en grec) ceux de G. A. Skinas, «Catalogue des cartes existantes » (de Rigas), et de Georges Tolias pour le colloque de Delphes (voir la note suivante). Bien entendu, il ne s'agit ici que d'une recension succincte.

5. «Grèce spacieuse : la carte de Rhigas et les limites de l'hellénisme » (en grec), Ta Istorika, vol. XV, fasc. 28-29, 1998.

6.Dans l'Histoire diplomatique de la Grèce de 1821 à nos jours (5 vol., Paris, 1925-1926), faite en collaboration avec Michel Lhéritier, l'ardent partisan de la Grande Idée que fut Édouard Driault se réfère fréquemment à la carte de Rigas, « la carte de toute son [la Grèce] histoire » (tome I, p. 465).

7.Nous avons toujours de grandes imprécisions sur sa vie, à part les trois dernières années. Concernant les circonstances de son arrestation par les Autrichiens et de son transfert aux mains des Ottomans, le fondateur des études néohelléniques françaises Émile Legrand a publié un important recueil de documents, Documents inédits concernant Rhigas Velestinlis et ses compagnons de martyre, Paris, 1892.

8.Il s'agit d'un " hymne de guerre », très célèbre en Grèce et encore chanté aujourd'hui. Fauriel l'a placé au début de ses Chants populaires de la Grèce moderne (1824).

9.Moniteur du 1er messidor an VI (19 juin 1798). Le journal parle de sa « passion presque délirante pour l'affranchissement de sa patrie ", et ajoute que "l'ancienne littérature de la Grèce échauffait son imagination ». Il est certain que les projets de Rigas manquaient de sérieux. Néanmoins, le Moniteur du 8 vendémiaire an VII (30 septembre 1798) écrit que « les Grecs semblent se réveiller de leur long sommeil».

10.Sous la dictature des colonels, des groupes d'étudiants contestataires ont pris son nom.

11. Voir le texte de cette constitution dans Notis Botzaris, Visions balkaniques de la préparation de la révolution grecque (1789-1823), Droz-Minard, 1962.

12.La constitution commence par : «Le peuple descendant des Hellènes... » 13.D'Anville s'explique longuement sur le mouvement de la géographie « actuelle et positive " pour " parvenir à fixer solidement le détail de l'ancienne géographie » (Analyse critique de la carte intitulée les côtes de la Grèce et de l'Archipel, 1757).

14.Sa vision de la Grèce, qui privilégie la virile Sparte aux dépens de l'Athènes décadente, a fortement influencé les révolutionnaires français. Voir Maurice Badolle, L'abbé J.-J. Barthélemy et l'hellénisme en France au xviiie siècle, Paris, 1926 ; un résumé de la vie et de l'œuvre de Barthélemy a été publié en 1996 par les Amis d'Aubagne (ville natale de Barthélemy) ; voir aussi la thèse de Jacques Bouineau, Réminiscences de l'Antiquité dans la Révolution française (1984), parue en 1986 sous le titre Les toges du pouvoir ou la Révolution de droit antique (Univ. Toulouse Le Mirail/Éd. Éché).

15.G. Tolias a publié un recensement des cartes grecques de Barbié du Bocage dans Kentron noehellenikon Eleunon, Athènes, 1993. Le Fonds Barbié du département des Cartes et Plans de la Bibliothèque nationale de France comprend une collection de 1415 cartes acquises en 1844. 1031 cartes sont de lui et 209 concernent la Grèce. Le Catalogue des livres de la bibliothèque de feu M. Barbié du Bocage (Paris, 1826), riche de 1134 titres, donne une haute idée de sa grande personnalité.

16.D. Philippides et G. Constantas, Neoterricae Geographia (« Géographie plus récente »), Vienne, 1791. 
17.La carte d'Antime Gazi, qui fait partie de la collection Barbié du Bocage et est entrée à la BNF en 1844, porte aux Cartes et Plans de la BNF la cote Ge DD 5963.

18.Elle fera d'immenses profits durant les années cruciales de la Révolution française, notamment en alimentant en céréales la France qui a alors perdu la maîtrise de la Méditerranée orientale.

19.Nous avons utilisé l'exemplaire de la carte entré aux Cartes et Plans de la BNF en 1847 (sous la cote CC 2656), et nous remercions chaleureusement le personnel du département pour l'aide constante qu'il nous a apportée, ainsi que Mme Mireille Pastoureau pour son accueil à l'Institut. Nos remerciements vont également à Geneviève Bluteau pour son aide dans la traduction des phrases de la carte.

20.1675-1726. Il est l'auteur d'une centaine de cartes qui, notamment à travers son gendre Buache, paraîtront jusque vers 1770 . Voir Numa Broc, La géographie des philosophes, université de Strasbourg, 1976.

21. Ces deux cartes sont au 1/1275000. Rigas a pu aussi utiliser la carte de Delisle « de la Hongrie et des pays qui en dépendaient autrefois » (1703) (BNF, Cartes et Plans, Ge D 11314). Pour les autres sources cartographiques de Rigas sur les parties au nord du Danube, voir plus loin.

22.Rigas a même copié le cartouche de la carte de Delisle, avec quelques changements (voir ci-après).

23.Philippidès et Gazi. Voir Correspondance, 1794-1819, Barbié du Bocage-Daniel PhilippidisAnthimos Gazis, éditée par Aikaterini Coumarianou, Athènes, 1966 (les lettres sont en français).

24.Meletius est un ecclésiastique (né à Janina en Épire en 1661 et mort en 1714). Sa Geographia palaia kai nea est parue à Venise en 1728. C'est une géographie qui se veut universelle, mais la Grèce y occupe 140 pages sur 600. De nombreuses phrases dont Rigas a parsemé sa carte viennent de là.

25.Grand Dictionnaire géographique, historique et critique, 6 volumes, 1768.

26. Voir par exemple Choiseul-Gouffier, Voyage pittoresque de la Grèce, tome I, Paris, 1782 (tome II, Paris, 1809).

27.Voyage du Jeune Anacharsis, tome II, chap. II.

28.Il y a çà et là sur la carte des phrases du type " patrie de... » ou « tombeau de... » (voir tableau en fin d'article). Il y a en outre de nombreuses monnaies, placées au hasard. Le tout accentue l'impression d'un espace densément occupé par les Grecs anciens. Les monnaies notamment créent de la continuité, alors qu'en réalité l'espace hellénique et les Balkans sont fortement morcelés.

29.Voir P. Grimal, Dictionnaire de la mythologie grecque et romaine. Relevons-y notamment ce qui concerne Hellen, fils de Deucalion et Pyrrha et ancêtre des Doriens, des Achéens, des Ioniens et des Éoliens. Pour Rigas, la Thessalie est bien le berceau de la Grèce et du monde hellénique.

30.Notation rare (il y a cependant aussi les monastères du mont Athos) : Rigas est hostile à l'orthodoxie. Après avoir soutenu les Lumières, l'Église orthodoxe s'est violemment élevée contre elles à partir de la Révolution française.

31. Cette barrière continue est parfaitement visible par exemple dans la Cosmographia de Sébastien Münster (1555).

32.Voir son « Essai de géographie physique où l'on propose des vues générales sur l'espèce de charpente du globe » (Mémoires de l'Académie des sciences, 1752).

33. C'est entre les années 1820 et 1840 que cette théorie commencera à être mise à mal. Même un grand spécialiste comme Ami Boué y recourt encore, dans son grand ouvrage 
sur la Turquie d'Europe (tome I, 1840). On y décèle son embarras, car il voit que la théorie ne cadre pas avec les observations. En fait, Boué est conscient qu'il existe des voies de passage dans cet espace si longtemps réputé infranchissable.

34.J.-Y. Guiomar, Correspondance G. Lejean-Ch. Alexandre, Librairie Touzot, 1993 M.-Th. Lorain, « Guillaume Lejean, voyageur-cartographe », Bulletin de la Société archéologique du Finistère, tome CXXIII, 1994.

35.La Bulgarie danubienne et le Balkan, 1881.

36.Voir notamment Vidal De La Blache, Géographie universelle, tome VII, Italie et pays balkaniques. Voir aussi Jovan Cvijic, La Péninsule balkanique, Armand Colin, 1918 ; Georges Prevelakis, Les Balkans, cultures et géopolitiques, Nathan, 1994.

37. Voir Ernoult et Maillet, Dictionnaire étymologique de la langue latine.

38.Voir G. Glotz, Histoire romaine, tome I, pp. 549-575 (d'après Polybe, Histoire générale, livres 29 à 36).

39.Voir G. I. Bratianu, Origines et formation de l'unité roumaine, Bucarest, 1943. Notons qu'en France la Dacie avait fait l'objet de trois communications de d'Anville en 1750 à l'Académie des inscriptions, La Dacie avant Trajan, La Dacie sous Trajan, La Dacie après Trajan.

40.La carte de Ruhedorf est aux Cartes et Plans de la BNF sous la cote Ge D 14654 (Mappa specialis Valachiae et (...) singulorum districtuum, 1788).

41.Praktika tès Akademias Athénon (1978). L'autographe de Rigas est sous la cote Ge F 5920.

42.Même si de nombreux Grecs, commerçants notamment, résident dans les Principautés et s'ils y forment l'élite intellectuelle, celle qui répand notamment les Lumières françaises.

43.Joseph Mesodace, ou Mesiodax, est l'un des grands hommes des lumières grecques, qui a entre autres publié une Théorie de la géographie, Vienne, 1781 (c'est donc l'une des inspirations possibles de Rigas) voir l'important ouvrage de C. Th. Dimaras, Histoire de la littérature néohellénique, coll. de l'Institut français d'Athènes, 1965.

44.Les guerres qui entre 1788 et 1792 ont opposé l'Empire ottoman à la Russie et à l'Autriche, et qui se sont conclues par les traités de Sistova (1791) et Iassi (1792). Les poussées russe et autrichienne vers les Balkans ont alors été bloquées.

45.Né à Smyrne en 1748 et d'abord médecin, il est surtout un grand éditeur de textes grecs anciens. Il vit à Paris de 1788 à sa mort en 1833. Il est notamment l'auteur d'un important Mémoire sur l'état actuel de la civilisation dans la Grèce, lu le 6 janvier 1803 à la Société des Observateurs de l'homme.

46.Sur ces questions, voir notamment Denis Kohler, « Naissance de l'historiographie grecque moderne », in Philologiques I, éditions de la MSH, 1990 ; Stella Manet, « C.

Paparrigopoulos et J. Michelet deux historiens romantiques, deux romantismes ", Storia della Storiografia, $\mathrm{n}^{\circ} 33$, 1998. La question est aussi abordée par J.-P. Vernant dans sa préface aux Mémoires du général Macriyannis (trad. D. Kohler, Albin Michel, 1986). Il note que le général emploie le nom d'Hellada quand il évoque les grands morts (" c'est le nom de notre patrie qui a disparu avec eux ») et parle des « Grecs » sur le plan de la langue.

47.Ouvr. cité, p. 306.

48.Rappelons qu'alors la langue était couramment appelée la « langue roméique ». 49.Id., p. 405. En italiques dans le texte. 
50.Voir André Mirambel, La France devant l'hellénisme, Paris, 1962, et C. Th. Dimaras, "Ambivalence de l'hellénisme ", Actes du 6e congrès de l'Association internationale de littérature comparée, pp. 557-560.

51.Voir J.-Y. Guiomar, « Histoire et significations de "la Grande Nation” (août 1797automne 1798) : problèmes d'interprétation », dans Du Directoire au Consulat. 1. Le lien politique local dans la Grande Nation, J. Bernet, J.-P. Jessenne, H. Leuwers éd., Lille 1999.

\section{RÉSUMÉS}

Le premier martyr de la cause protonationale grecque, Rigas Velestinlis, exécuté à Belgrade par les Ottomans en 1798, a réalisé en 1797 à Vienne la première carte moderne en grec de la Grèce. Cette carte est un manifeste politique, où Rigas tente de visualiser l'espace sur lequel il veut créer un État hellénique à la fois unitaire et supranational, comprenant tous les Balkans, les principautés danubiennes et la Grèce d'Asie mineure. La mise en évidence du caractère hellénique de cet énorme espace se fait par le recours à l'histoire : la carte dresse l'état des lieux où la présence grecque est attestée par la mythologie et l'histoire depuis les temps les plus anciens. À l'heure où les Habsbourg et les Russes affirment leurs prétentions à la succession de l'Empire ottoman, Rigas, encouragé par la présence française dans les îles Ioniennes, annonce la candidature de la Grèce à cette succession.

\section{Rigas's Map of Greece and the Name of Greece}

The first martyr of the protonationalist Greek cause, Rigas Velestinlis, executed in Belgrade by the Ottomans in 1798, drew up the first modern map of Greece in Greek at Vienna in 1797. This map is a political manifesto, in which Rigas attempted to visualize the space in which he yearned to create an Hellenic state both unified and supranational, comprising all the Balkans, the Danube princedoms and the Greek lands of Asia Minor. The Hellenic character of this vast territory is underscored by historical reference, the map showing all the places where a Greek presence is attested by mythology or history since the earliest times. At a period when the Hapsburgs and the Russians were asserting their claims to the succession of the Ottoman Empire, Rigas, encouraged by the presence of the French in the Ionian islands, announced that Greece too was a candidate to this succession.

\section{La carta della Grecia e il nome della Grecia}

Il primo martire della causa nazionale greca, Rigas Velestinlis, giustiziato a Belgrado dagli Ottomani, ha realizzato nel 1797 a Vienna la prima carta moderna della Grecia. Questa carta è un manifesto politico nel quale Rigas tenta di visualizzare lo spazio sul quale vuole creare uno Stato ellenico nello stesso tempo unitario e supranazionale che includa gli interi Balcani, i principati danubiani e la Grecia d'Asia Minore. La messa in evidenza del carattere ellenico di quell'enorme area avviene mediante la storia: la carte elenca i luoghi dove la presenza greca è attestata dalla mitologia e dalla storia dai tempi più remoti. Nell'ora in cui gli Asburgo e i Russi ribadiscono le loro pretese alla successione dell'Impero Ottomano, Rigas, incoraggiato dalla presenza francese nelle isole ionie, annuncia la candidatura della Gecia a questa successione.

El primer mártir de la causa protonacionalista griega, Rigas Velestinlis, matado en Belgrado por los otomanes en 1798, realizó en Viena la primera mapa de Grecia en Griego. Este mapa aparece 
como un manifiesto político, donde Rigas intenta una visualización del espacio donde quiere crear un estado helenístico a la vez unitario, supranacional, incluyendo los Balkans, los principados danubianos y la Grecia de Asia menor. El carácter helenistico de este importante espacio se justifica por la historia: el mapa identifica los sitios donde la presencia griega esta atestada por la historia y la mitología desde los tiempos los más antiguos. Incitado por la presencia francesa en las islas jonias, Rigas afirma las pretensiones de Grecia cuando los Habsbourg y los rusos pretenden a la sucesión del imperio otoman.

Rigas' Karte von Griechenland und der Name Griecheliand.

Der erste Märtyrer der protonationalen griechischen Sache, Rigas Velestinlis, der 1798 in Belgrad von den Ottomanen erschossen wurde, hat 1797 in Wien die erste moderne in Griechisch verfaßte Karte Griechenlands verfertigt. Diese Landkarte ist ein politisches Manifest, wo Rigas versucht, den Raum sichtbar zu machen, auf dem er einen hellenischen, einheitlichen sowie supranationalen Staat gründen will: Dieser soll den ganzen Balkan, die Donaufürstentümer und das Griechenland Kleinasiens einschließen. Um den hellenischen Charakter dieses Riesenraums offenbar zu machen, bedient sich Rigas der Geschichte: Seine Karte verzeichnet alle Orte, wo die Anwesenheit der Griechen seit den ältesten Zeiten durch die Mythologie oder die Geschichte bezeugt ist. $\mathrm{Zu}$ einer Zeit, als die Habsburger und die Russen Anspruch auf die Erbschaft des ottomanischen Reiches erhehen, zeigt Rigas, durch die französische Präsenz auf den ionischen Inseln ermutigt, daß sich auch Griechenland um diese Erbfolge bewirbt. 